bezeichnen, um eine feindliche Stadt zu er- Diese Schreibung beweist, dass als Stamm der

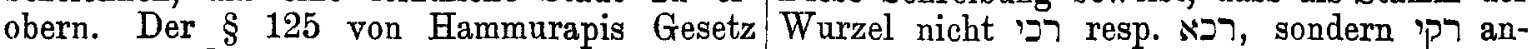
zeigt aber, dass auch in ein Privathaus ver- zusetzen ist. Ob er mit arab. قو zusammenmittelst pilšu und nabalkattu ein Einbruch verübt hängt, erscheint mir fürs erste noch unsicher. werden kann. Leider ist der von Scheil DEP $X 83$ publizierte, von Ungnad als $\S 76$ gezählte Paragraph des Gesetzbuches nicht gut erhalten; aber auch hier werden nabalkattu und palâšs nebeneinander erwähnt. Da nun nabalkutu zweifellos die Bedeutung „übersteigen, hinübersteigen" hat (z. B. CT XVI, 14, 54: ana uriina ai ibbalkitûni = auf mein Dach mögen sie nicht hinübersteigen; Maklû II, 154: kîma pitikti ana nabalkutiia = um mich wie eine Lehmmauer zu übersteigen), so bedeutet nabalkattı „das Hinübersteigen, Einsteigen“ oder das Instrument zum Hinübersteigen d. i. "die Leiter" (so vermutete schon Jensen bei Schrader, Sendschirli I, 43). Das Haus wird also durch ein Loch in der elenden Lehmmauer oder durch Einsteigen ausgeraubt, die feindliche Stadt durch Breschelegen und Leitern erobert. Eine Illustration der Einnahme einer Stadt durch pilšu und nabalkattu bietet das beiLa yard, Mon. I, 20 publizierte Relief Assurnassirpals, wo rechts unten ein assyrischer Krieger mit seinem Schwerte ein grosses Loch in die Mauer der feindlichen Stadt gebohrt hat, während daneben ein anderer Soldat sie auf einer hohen Leiter übersteigt.

\title{
3. nark̂k.
}

Die Bedeutung "Zuflucht" war für das Wort markitu schon seitlangem erwiesen; s. Delitzs ch HW 619. Daneben kommt auch die Form nirkîtu (Thureau-Dangin, $8^{\text {me }}$ camp. deSargon Z. 255) vor. Das zu diesen Substantiven gehörige Verbum ist nunmehr auch in mehreren Formen nachgewiesen worden. In I, 2 bedeutet es „Zuflucht gewähren"; vgl. CH. VIII, 43: wardam .... halgam ... ina bitišu ir-ta-li $i=$ einem entflohenen .... Sklaven .... hat er in seinem Hause Zuflucht gewährt. Nicht ganz klar ist die Bedeutung bei $\mathrm{Knudtzon,} \mathrm{Amarna}$ Nr. 161, 32: ti-ir-ta-ki-i-mi=du bast dich zurückgezogen(?). narkîu (IV, 1) hat die Bedeutung "sich flüchten, seine Zuflucht nehmen"; vgl. Ebeling, Assur Nr. 31 Rs. 16: ana zikir šumešunu na-ar-ki $=$ auf die Nennung ibres Namens flüchte. Das Ideogramm ist $H A-A$, das sonst $=$ halâk $u$, na'butu ist. Das neuerdings von Clay, Yale University Coll. I, 85 ff. publizierte Vokabular bietet Kol. I, 13 folgende Gleichungen :

$\ldots \ldots=\left[T Y\left\langle\langle\rangle=\prod\right.\right.$ d. i. ša-aialıu $]-k u-\dot{u}-a-$ II d. i. $\cdot i-g u b=h a-l a-k i n c ; n a-b u-t u ; n a r-k u-u u^{1}$. $71,126$.

${ }^{1}$ Korrekturzusatz: Vgl. jetzt auch Ungnad ZDMG

\section{Zur Serie harra = hubullu.}

Von A, Schollm eyer.

Als weiteres Duplikat zur III. Tafel der Serie harra = hubullu ist ausser den von Meissner und Ungnad (OLZ 1915, 136; 1917, 73) besprochenen Texten das von Scheil, Recueil de travaux Vol. XXXVI (S. 12 des Separatabzuges) veröffentlichte Fragment zu nennen, das bereits von Scheil als Duplikat zu Rm. 367 erkannt wurde. Mit Kol. II, Z. 41 nach der Zählung Meissners (MVAG 1913, 2 S. 18) setzt das anscheinend zweikolumnige Fragment ein und bricht mit Z. 58 des Textes ab.

Die bemerkenswerten Varianten und Abweichungen sind bereits von Scheil verzeichnet. In Z. 44 scheint [gišs]-ḩa-šur = sap-[pan-du ${ }^{1}$ zu sein, obwohl Scheil keine Ergänzung wagt. Beachtenswert ist ferner giš-kib-gal = ha-ah[hu], das sonst auch kameššeru entspricht. In der vorletzten Zelle bietet das Duplikat [gišl] am-ga[r = bu-tu-ut-tu]; vielleicht ist das unsichere gar mit Meissner Kol. II, Z. 57 gal zu lesen.

\section{Reste der Sprache von Hanigalbat? \\ Von Otto Schrooder.}

Neben den zahlreichen sumerisch-akkadischen Vokabularen haben sich eine geringe Anzahl von Vokabularbruchstücken erhalten, diezweifellos Unsumerisches Sprachgut behandeln, ohne dass wir bisher in der Lage wären, dieses Sprachgut näher zu bestimmen. Ich erinnere an VR $20 \mathrm{Nr}$. 4, wo wir Z. 57-60 die Gleichungen lesen:

$$
\begin{aligned}
& k u=a-n a-k u \\
& l i=a t-t a \\
& \check{s} e=\check{s} \dot{u}-\hat{u} \\
& \grave{s} i=s ̌ u-a-t u m
\end{aligned}
$$

(Vgl. dazu Delitzsch, Sumerische Grammatik S. 24, Anm. 1). Etwas umfangreicher ist das Stück VR 27, $31 \mathrm{ff}$; dort finden wir zunächst in Zeile $34-37$ folgende Gleichungen:

$$
\begin{aligned}
& l i u=a-n a-[k i u] \\
& k u=a t-t a \\
& l a=s i \hat{\imath}-\hat{\imath} \\
& l i=s s i-a-t u m .
\end{aligned}
$$

Man beachte, dass in beiden Stücken die Anordnung der akkadischen Spalte die gleiche ist; es handelt sich um zwei verschiedene Sprachen;

- Zu sappandu $=$ Senf (sinapis alba) vgl. Holma, Kleine Beiträge zum assyr. Lexikon S. 78. 
die des zweiten Fragments lässt sich vielleicht bestimmen.

Hierzu ist die elfzeilige Inschrift des Agabtaha verwertbar, die Scheil in "Délégation en Perse, Mémoires Vol. II (Textes élamites-sémitiques I) ${ }^{\prime}$ S. $95 \mathrm{f}$. und pl. 20 mitteilte. Sie stammt von einem Manne namens $A$-ga-ab-ta-ha, der unter Kaštiliaš aus Hanigalbat entfloh und am Hofe dieses Königs Zuflucht fand; er nennt sich in seiner Inschrift mu-un-na-bi-it-tum $\forall a-$ li-gal-ba-tu-u "Hanigalbatäischer Flüchtling", legt also merkwürdigerweise auf die Tatsache seiner Flucht aus dem Vaterlande gewissen Nachdruck, wie es etwa die französischen Réfugiés taten.

Bei Besprechung unserer Inschrift sagt Winckler (OLZ $1901 \mathrm{Sp} .451=$ Kritische Schriften II S. 97): "Merkwürdig ist die Schreibung $\forall a-l i-g a l-b a-t u-u$. Mit Scheil muss man daraus zunächst die Lesung $l i$ des $n i$ der gewöhnlichen Schreibung folgern, aber in TelAmarna wird es einmal mit na geschrieben, so dass wohl hier eine schlechte Schreibung oder ein Versehen anzunehmen ist." Da wir bisher nur die Schreibungen Ha-ni-gal-bat, Hana-kal-bat (VAT $333,10=$ VAS XI Nr. 146) fanden, ist eire Lesung mit $l$ statt $n$ einfach unmöglich, wenngleich man sich hin und her unter dem Einfluss des Agabtaha-Textes zu deren Annahme bewogen fïhlte. - Nun wäre noch eine dritte Möglichkeit da, dass es sich nicht um eine "schlechte" Schreibung handelt, sondern dass der Inschriftschreiber sich besonders fein ausdrücken wollte; das scheint in der Tat so gewesen zu sein.

Man beachte nämlich, dass sich VR 27, $43 \mathrm{ff}$. für $L I$ folgende akkadische Werte finden: $i-n a$,

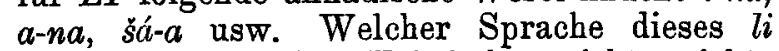
angehört, sagt das Vokabular nicht; nichts schliesst daher die Annahme aus, dass es sich um ein Wort der Sprache von Hanigalbat handelt. Agabtaha, der ,hanigalbatäische Flüchtling" schreibt in seiner Inschrift Babylonisch, aber er erlaubt sich die Freiheit, in ihr $L I$ mit dem Lautwert ana za benützen, weil das hanigalbatäische $L I$ einem akkadischen ana entspricht. Wenn ein voller Beweis für meine Vermutung, dass VR 27 ein hanigalbatäisch-akkadisches Vokabular ist, hierdurch nicht geliefert ist, so mag man doch die Möglichkeit der Erklärung für später im Auge behalten.

\section{Zur Etymologie von}

Von P. S. Landersdorfer.
ZDMG 29 , S. 145 , Bedjan, Homiliae selectae Mar Jacobi Sarugensis, tom. 3, S. 795) Vers 62 der Ausdruck أبح| יمتر. Das Wort i scheint sonst im Syrischen noch nicht belegt zu sein, wenigstens von den mir zu Gebote stehenden Lexica führt es Brun überhaupt nicht auf, während Brockelmann lediglich unsere Stelle zitiert. Aber auch die Etymologie des Wortes bietet Schwierigkeiten, da sich weder im Syrischen noch in den übrigen semitischen Sprachen eine entsprechende Wurzel zu finden scheint.

Die Bedeutung lässt sich leicht aus dem Zusammenhang festlegen. Schon der Zusatz متتر deutet darauf hin, dass es sich höchstwahrscheinlich um ein Wasserbehältnis handelt. Da sich ferner die in Betracht kommende Stelle auf den heidnischen Kult von Mabug-Hierapolis bezieht und wir anderweitig wissen, (Lukian, de dea Syra 10 u. 32), dass im Kult der Atargatis der heilige Quellteich eine Hauptrolle

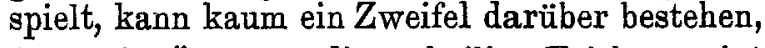
dass mit ist (vgl. meine Schrift: Die Götterliste des Mar Jacob von Sarug in seiner Homilie über den Fall der Götzenbilder, S. 51 f.).

In Ermangelung einer befriedigenden semitischen Etymologie möchte ich die Vermutung aussprechen, dass $\mathrm{h}^{\mathrm{i}}$ sumerischen Ursprungs ist. Zunächst möchte man an egâa "Flut, Hochflut" denken (Delitzsch, Sum. Glossar S. 30), aus a-gê-a (TY<EETY), wobei dann, da eine direkte Entlehnung aus dem Sumerischen doch kaum anzunehmen ist, das akkadische agû (Delitzsch, HWB S. 15) die Vermittlerrolle gespielt haben müsste. Doch hat diese Annahme wegen des a-Lautes einige Schwierigkeit, da nicht einzusehen ist, warum im Syrischen ein i-Laut daraus hätte werden sollen. Ich möchte darum eher an $e(g)$ "Graben, Kanal" denken, das als $i k u$ in das Akkadische übergegangen ist (Delitzsch, Glossar, S. 29), welch letzteres ohne Schwierigkeit in syrisch kennen ist.

\section{Eine Mond- und Sonnenfinsternis im Alten Testament.}

Von Wilhelm Erbt.

Im Hesekielbuche steht eine Sammlung von Aussprüchen gegen den König von Aegypten. Jeder von ihnen wird durch ein Datum eingeleitet. Jeder von ihnen ist mit einer Deutung versehen worden, die ihn auf die Zeit Nebukadnezars bezieht. Bereits Winckler hat den

Bei Jakob von Sarug findet sich in der Nachweis geführt, dass Apries und Amasis, Homilie tiber den Fall der Götzenbilder (Martin, die Pharaonen dieser Tage, nicht in Be- 